\title{
Deficiência auditiva neurossensorial associada à doença de Kawasaki
}

\author{
Sensorineural hearing loss associated with Kawasaki disease
}

\author{
Carlos H.M. da Silva ${ }^{1}$, Isabel C.R.G. Roscoe ${ }^{2}$, Karla P. Fernandes ${ }^{3}$, Ricardo M. Novaes ${ }^{4}$ Carolina S. Lázari $^{5}$
}

\section{Resumo}

Objetivo: a doença de Kawasaki (DK) é uma vasculite sistêmica idiopática, autolimitada, de pequenos e médios vasos. Foram descritos na literatura treze casos de deficiência auditiva neurossensorial, durante a evolução dessa doença. Descreve-se o caso de um lactente com doença de Kawasaki com evolução, durante a fase aguda, para deficiência auditiva neurossensorial. A descrição do caso justifica-se por ilustrar uma complicação grave da DK, pouco mencionada na literatura, que reforça a recomendação de cautela na avaliação global destes pacientes.

Descrição: menino hígido de 1 ano e sete meses de idade apresentou-se com febre diária persistente, iniciada havia sete dias, associada à irritabilidade, conjuntivite bilateral não-exsudativa, eritema máculo-papular em tronco. Evoluiu com artrite em punhos, cotovelos, joelhos e tornozelos e edema e descamação de mãos e pés, que impossibilitava a deambulação. O diagnóstico de doença de Kawasaki foi estabelecido de acordo com os critérios da American Heart Association (AHA), e a criança foi tratada de forma convencional. Houve regressão das manifestações clínicas três dias após o início do tratamento. Contudo, um mês após o início dos sintomas, os familiares notaram resposta insatisfatória a estímulos sonoros. A avaliação da acuidade auditiva através do BERA (Brainstem Evoked Responses Audiometry - Audiometria de Potenciais Evocados de Tronco Cerebral) diagnosticou perda auditiva neurossensorial bilateral, grau severo a profundo.

Comentários: é possível que o exame rotineiro de acuidade auditiva em crianças com doença de Kawasaki possa identificar precocemente a deficiência auditiva neurossensorial.

J Pediatr (Rio J) 2002; 78 (1): 71-74: vasculite, síndrome do linfonodo mucocutâneo, perda auditiva sensorioneural.

1. Adjunto-doutor do Depto. de Pediatria da Faculdade de Medicina da Universidade Federal de Uberlândia.

2. Médica do Depto. de Pediatria da Faculdade de Medicina da Universidade Federal de Uberlândia.

3. Mestre em Clínica Médica pela Faculdade de Medicina da Universidade Federal de Uberlândia.

4. Mestre em Otorrinolaringologia pela Escola Paulista de Medicina da Universidade Federal de São Paulo.

5. Aluna do $5^{\circ}$ ano da Faculdade de Medicina da Universidade Federal de Uberlândia.

Artigo submetido em 09.07.01, aceito em 31.10.01.

\begin{abstract}
Objectives: Kawasaki disease is a systemic idiopathic selflimited vasculitis of small and medium-sized vessels. Thirteen cases of sensorineural hearing loss during the evolution of this disease have been described in the literature. We describe a case of an infant with Kawasaki disease who developed sensorineural hearing loss during the acute phase. This case report shows a complication of Kawasaki disease, with few citations in literature, enhancing the importance of careful evaluation of these patients.

Description: nineteen-month-old-boy, formerly healthy, with persistent daily fever seven days before admission, associated with irritability, bilateral nonexudative conjunctivitis and maculopapular erythema on his trunk. There was later development of arthritis on wrists, elbows, knees, and ankles, and swelling associated with desquamation of hands and feet, which prevented him from walking. The diagnosis of Kawasaki disease was established according to the American Heart Association's criteria, and the child was conventionally treated. There was regression of clinical manifestations three days after the beginning of treatment. However, one month after the onset of symptoms, the family noticed an unsatisfactory response to sound stimuli. The evaluation of auditory acuity through BERA (Brainstem Evoked Responses Audiometry) revealed severe bilateral sensorineural hearing loss.

Comments: routine examination of auditory acuity in children with Kawasaki disease may help identify sensorineural hearing loss at an early stage.
\end{abstract}

JPediatr (Rio J) 2002; 78(1): 71-74: vasculitis, mucocutaneous lymph node syndrome, sensorineural hearing loss.

\section{Introdução}

A doença de Kawasaki (DK) é uma vasculite sistêmica idiopática, autolimitada, de pequenos e médios vasos ${ }^{1}$. A manifestação determinante do prognóstico da doença é a vasculite coronariana, que acomete cerca de $25 \%$ dos pacientes não tratados ${ }^{2}$. Embora o comprometimento grave do sistema nervoso central seja infreqüente, foram descritos na literatura treze casos de deficiência auditiva neuros- 
sensorial, durante a evolução dessa doença ${ }^{3,4}$ (Tabela 1). Descreve-se o caso de um lactente com doença de Kawasaki com evolução, durante a fase aguda, para deficiência auditiva neurossensorial. O objetivo dos autores com esta descrição é ilustrar uma complicação grave da DK, pouco mencionada na literatura, que reforça a recomendação de cautela na avaliação global destes pacientes.

\section{Descrição do caso}

Menino hígido de 1 ano e sete meses, procedente da cidade de Uberlândia-MG, apresentou-se com febre diária persistente, iniciada havia sete dias, associada à irritabilidade, conjuntivite bilateral não-exsudativa, eritema máculopapular em tronco. Evoluiu com artrite em punhos, cotovelos, joelhos e tornozelos e edema e descamação de mãos e pés, que impossibilitavam a deambulação. A investigação laboratorial revelou: $\mathrm{Hb}=11,0 \mathrm{~g} / \mathrm{dl}$; leucócitos $=7600 / \mathrm{mm}^{3}$; plaquetas $=161000 / \mathrm{mm}^{3}$; velocidade de hemossedimentação $=55 \mathrm{~mm} / \mathrm{h}$, proteína-C-reativa $=24 \mathrm{mg} / \mathrm{l} ; \mathrm{ASLO}=50 \mathrm{UI}$; albumina $=4,2 \mathrm{~g} / \mathrm{l}$; sorologias para toxoplasmose, mononucleose, rubéola e citomegalovírus negativas. Ecocardiogramas e eletrocardiogramas seriados não apresentaram anormalidades.

O diagnóstico de doença de Kawasaki foi estabelecido de acordo com os seguintes critérios da American Heart Association $(A H A)^{5,6}$ : presença de febre persistente por pelo menos cinco dias; alterações de extremidades, tais como eritema e edema de mãos e pés na fase aguda, e descamação membranosa de polpas digitais na fase de convalescença; exantema polimórfico; conjuntivite nãoexsudativa bilateral indolor; alterações em lábios e cavidade oral, tais como eritema e fissuras em lábios, "língua em framboesa" e hiperemia difusa em mucosa orofaríngea. Destes, o paciente descrito preenchia os quatro primeiros critérios, caracterizando-se, portanto, como Kawasaki incompleto.

A criança foi tratada de forma convencional com gamaglobulina endovenosa ( $2 \mathrm{~g} / \mathrm{kg} / \mathrm{dia})$ e aspirina $(100 \mathrm{mg} / \mathrm{kg} / \mathrm{dia}$ até o $14^{\circ}$ dia de doença). Houve regressão das manifestações clínicas três dias após o início do tratamento. Contudo, a criança permaneceu com dificuldade para deambular, embora houvesse regressão do quadro articular e, um mês após o início dos sintomas, os familiares notaram resposta insatisfatória a estímulos sonoros. A tomografia craniana e de ossos temporais não evidenciaram anormalidades.

A avaliação da acuidade auditiva foi realizada através do BERA (Brainstem Evoked Responses Audiometry Audiometria de Potenciais Evocados de Tronco Cerebral), uma vez que este é o exame indicado para avaliação audiológica de recém-nascidos e lactentes, que ainda não têm capacidade de colaborar para a realização de uma audiometria tonal convencional. O exame consiste no registro das respostas elétricas desencadeadas por um estímulo sonoro ao longo da via auditiva até o tronco cerebral. A

Tabela 1- Resumo dos casos de doença de Kawasaki, com perda auditiva neurossensorial, descritos na literatura

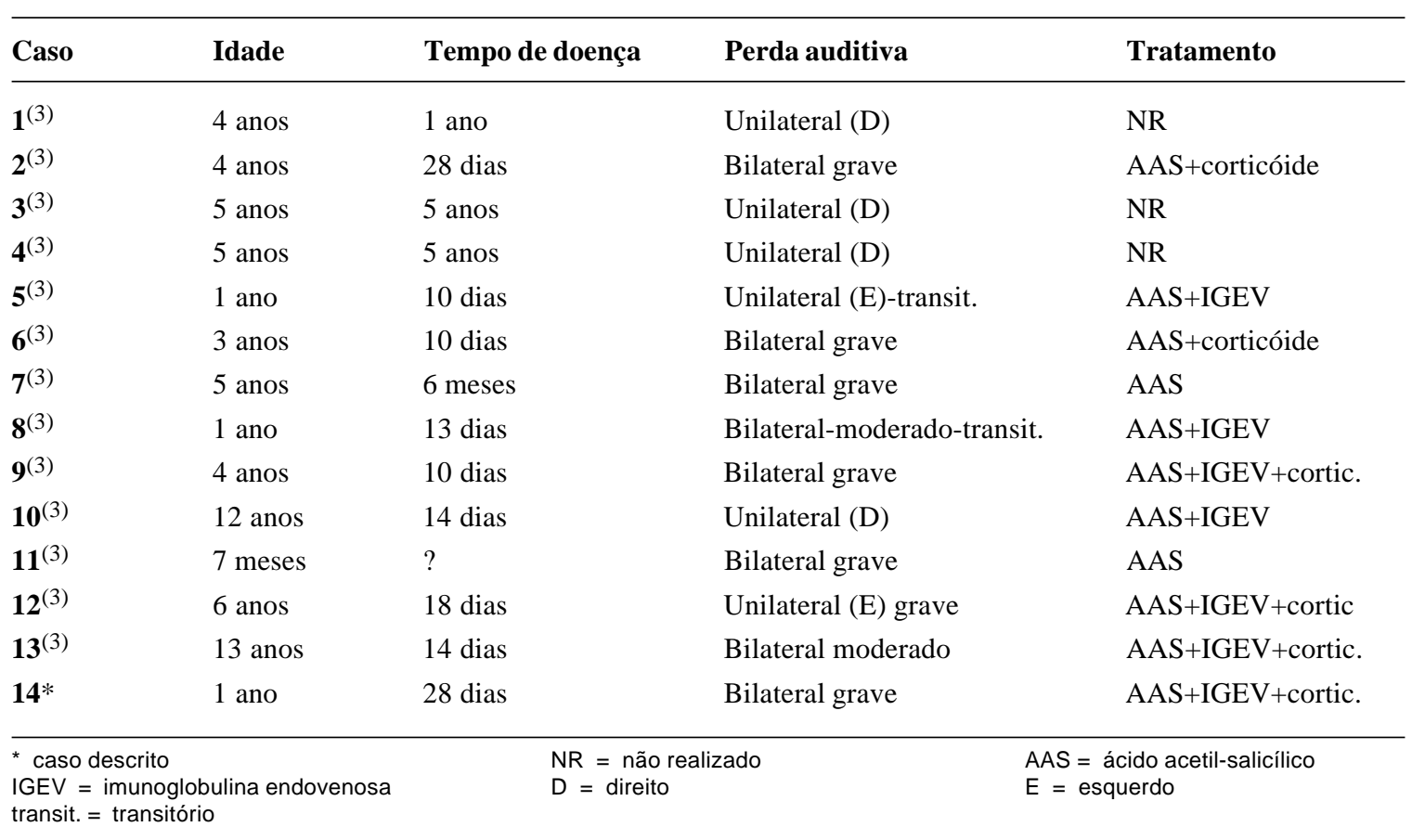


interpretação é feita com base no potencial de ação, que se origina no nervo coclear (NC VIII) e nas vias auditivas, e sobe pelo tronco cerebral. $\mathrm{O}$ traçado normal registra seis ondas: I, II, III, V, VI e VII, originadas, respectivamente, na porção do NC VIII distal ao tronco cerebral, na porção do NC VIII proximal ao tronco cerebral, no núcleo coclear, no complexo olivar superior, no colículo inferior e no corpo geniculado medial. $\mathrm{O}$ exame do paciente descrito mostrou ausência de ondas diante de estímulos deflagrados a $90 \mathrm{~dB}$, em ambas as orelhas, o que sugere perda auditiva neurossensorial bilateral, grau severo a profundo. Apesar do tratamento com predinisona (dose inicial de $2 \mathrm{mg} / \mathrm{kg} / \mathrm{dia}$ ) por dois meses, não houve melhora da acuidade auditiva.

\section{Discussão}

As principais complicações da DK são relacionadas ao comprometimento cardíaco, em especial à vasculite coronariana $^{2,7,8}$. O caso descrito ilustra a existência de outro tipo de complicação grave e irreversível, o déficit auditivo neurossensorial, pouco mencionado na literatura ${ }^{3,4}$. Com efeito, a deficiência auditiva percebida durante a fase subaguda de um lactente com DK reforça o conceito de que esta é uma doença inflamatória sistêmica e recomenda-se, portando, cautela na avaliação global destes pacientes, pois teoricamente a lesão vascular pode estar presente em qualquer tecido humano.

Embora seja comum na fase aguda febril da DK a presença de extrema irritabilidade, possivelmente decorrente de meningite asséptica, as manifestações neurológicas nesta doença são raras ${ }^{8}$. Convulsões, comprometimento de pares cranianos (sétimo par) e hemiparesia causada por infartos ou tromboses são relatados na literatura ${ }^{1,3,4}$.

Até o presente, foram descritos na literatura apenas treze casos de perda auditiva neurossensorial associada à doença de Kawasaki ${ }^{3,4}$. Entretanto, esse número parece subestimado, pois a diminuição da acuidade auditiva nesta doença pode ser discreta e transitória e apenas detectada através de audiometria ou, em crianças menores, do BERA. Assim como no caso relatado, a deficiência auditiva geralmente é grave, bilateral e permanente, mas pode ser unilateral e transitória. Embora a maioria dos pacientes com esta complicação tenha sido tratada com aspirina em doses antiinflamatórias, é pouco provável que a deficiência auditiva tenha sido relacionada a esta medicação, pois o seu nível sérico, quando determinado, permaneceu sempre abaixo de $20 \mathrm{mg} / \mathrm{dl}$. Além disso, ao contrário do que ocorre na ototoxicidade associada à aspirina, a deficiência auditiva não foi transitória na maioria dos pacientes relatados.

O tempo entre o diagnóstico de DK e a percepção do déficit auditivo varia, nos casos relatados, entre 10 dias e 5 $\operatorname{anos}^{3,4}$. Essa demora parece correlacionar-se com a dificuldade da definição deste diagnóstico em crianças menores, principalmente abaixo de dois anos de idade, quando a percepção dos pais pode falhar, e a audiometria convenci- onal nãoé possível de ser realizada. No caso descrito, talvez dois fatores certamente contribuíram para que esta percepção tenha sido tardia: a persistência da irritabilidade além da primeira semana da doença e o fato de que a criança tinha adquirido apenas recentemente uma linguagem oral mais elaborada.

Alguns pacientes com perda auditiva neurossensorial associada à DK apresentaram outras manifestações neurológicas, como paralisia facial e ataxia ${ }^{3,4}$. No caso descrito, observou-se quedas freqüentes atribuídas inicialmente às manifestações articulares dolorosas e, posteriormente, ao comprometimento vestibular, pela presença de tonturas.

Várias anormalidades imunológicas são verificadas nos pacientes com $\mathrm{DK}^{8}$. Ocorre inicialmente ativação das células endoteliais vasculares e de moléculas de adesão induzidas por citocinas associadas ao aumento da porcentagem de células $\mathrm{CD} 4$, à diminuição de células $\mathrm{CD} 8$ positivas ativadas, e ao aumento da produção de imunoglobulinas pelas células B. O mecanismo fisiopatológico responsável pela vasculite no ouvido médio, nos pacientes com DK e com perda auditiva neurossensorial, é desconhecido. É possível que a lesão se localize nos vasos cocleares ou vasa nervorum, resultando no comprometimento do nervo acústico e vestibular.

O tratamento convencional para a DK (aspirina e gamaglobulina endovenosa) e, em alguns casos, como na presente descrição, a corticoterapia oral ou parenteral não alteraram a evolução da perda auditiva ${ }^{8,9}$. É possível que a lesão do ouvido interno ocorra nos primeiros dias da doença, ou seja, na fase febril inicial, antes da administração do tratamento específico, normalmente realizado entre o quinto e décimo dia da doença, quando o diagnóstico pode ser definido através dos critérios estabelecidos. É provável que o tratamento precoce - durante a primeira semana da doença - com gamaglobulina endovenosa e/ou corticosteróides possa contribuir para evitar esta grave complicação.

Sundel et al. ${ }^{4}$ sugerem a necessidade de pelo menos uma avaliação audiológica durante o seguimento de todos os pacientes com diagnóstico de doença de Kawasaki, uma vez que surdez neurossensorial transitória ou persistente pode ocorrer em pacientes com esta doença, especialmente naqueles com febre prolongada e com marcadores laboratoriais sugestivos de inflamação sistêmica grave.

Concluindo, é possível que o exame rotineiro de acuidade auditiva em crianças com doença de Kawasaki possa identificar precocemente a deficiência auditiva neurossensorial.

\section{Referências bibliográficas}

1. Cassidy JT, Petty RE. Vasculitis. In:__ Textbook of Pediatric Rheumatology. Philadelphia: WB Saunders; 1995. p.365-422.

2. Kato H, Sugimura T, Akagi T, et al. Long-term consequences of Kawasaki disease: a 10- to 21-year follow-up study of 594 patients. Circulation 1996;94:1379-85. 
3. Sundel RP, et al. Sensorineural hearing loss associated with Kawasaki disease. J Pediatr 1990;117:371-7.

4. Sundel RP, et al. Audiologic profiles of children with Kawasaki disease. Am J Otol 1992; 13:512-5.

5. Dajani AS, Taubert KA, Gerber MA, et al. Diagnosis and management of Kawasaki disease in children. Circulation 1993;87:1776-80.

6. Dajani AS, Taubert KA, Takahashi M, et al. Guidelines for longterm management of patients with Kawasaki disease: report from the Committee on Rheumatic Fever, Endocarditis, and Kawasaki Disease, Council on Cardiovascular Disease in the Young, American Heart Association. Circulation 1994;89:916-22.

7. Laupland KB, Dele Davies H. Epidemiology, etiology, and management of Kawasaki disease: state of the art. Pediatr Cardiol 1999; 20: 177-83.

8. Leung DY, Schlievert PM, Meissner HC. The immunopathogenesis and management of Kawasaki syndrome. Arthritis Rheum 1998; 41:1538-47.
9. Newburger JW, Takahashi M, Beiser AS, et al. A single intravenous infusion of gamma globulin compared with four infusions in the treatment of acute Kawasaki syndrome. N Engl J Med 1991;324:1633-9.

Endereço para correspondência:

Dr. Carlos Henrique Martins da Silva

Av. Rondon Pacheco, 3333 - apto. 103

CEP 38400-020 - Uberlândia, MG

Fone: (034) 235.0072 - Fax: (034) 235.0072

E-mail: carloshm@nanet.com.br 